hep-ph/9912471

DSF $41 / 99$

\title{
Unstable Heavy Majorana Neutrinos and Leptogenesis
}

\author{
Gianpiero Mangano and Gennaro Miele \\ Dipartimento di Fisica, Universitá di Napoli "Federico II", and INFN, Sezione di Napoli, \\ Mostra D'Oltremare Pad. 20, I-80125 Napoli, Italy
}

\begin{abstract}
We propose a new mechanism producing a non-vanishing lepton number asymmetry, based on decays of heavy Majorana neutrinos. If they are produced out of equilibrium, as occurs in preheating scenario, and are superpositions of mass eigenstates rapidly decaying, their decay rates contains interference terms provided the mass differences $\Delta m$ are small compared to widths $\Gamma$. The resulting lepton asymmetry, which is the analogue of the time-integrated $C P$ asymmetry in $B^{0}-\bar{B}^{0}$ system, is found to be proportional to $\Delta m / \Gamma$.
\end{abstract}

PACS number(s): 98.80.Cq; 98.80.-k; 14.60.P 


\section{Introduction}

A possible mechanism leading to the production, in the early universe, of a baryonantibaryon asymmetry can be found in terms of lepton number production by heavy Majorana neutrino decays [1]. As well known, the lepton number $L_{0}$ so generated is reprocessed by sphaleron transitions, and partially converted into baryon number B

$$
B=-\frac{8 n_{g}+4 n_{H}}{22 n_{g}+13 n_{H}} L_{0}
$$

where $n_{g}$ is the number of fermion generations, and $n_{H}$ denotes the number of Higgs doublets of electroweak Standard Model. There are two crucial issues in this mechanism which eventually determine its efficiency in producing the value for the baryon to photon number $\eta \sim\left(10^{-10} \div 10^{-9}\right)$, fixed by observations on light nuclei abundances.

First of all, the number density of heavy right-handed Majorana neutrinos $N_{i}$ depends on the mechanism of reheating. Since the produced lepton number is proportional to the number of $N_{i}$ per comoving volume, it is quite important to have a prediction for this parameter, as function of neutrino masses. This has been considered by many authors in the usual scenario of neutrino production via thermal excitations in the bath after reheating. In this case neutrino masses should be smaller than the maximal temperature obtained during the reheating of the universe. It has been recently shown by following in detail the reheating mechanism [2] that a reasonable estimate for this upper bound is of the order of $10^{3} T_{R H}$, where $T_{R H} \simeq \sqrt{\Gamma m_{P l}}$, is the so-called reheating temperature, $\Gamma$ being the decay rate of the inflaton. The value of $T_{R H}$ is constrained to be in the range $\left(10^{8} \div 10^{10}\right) \mathrm{GeV}$ in order to avoid an overproduction of gravitinos in SUSY scenarios [3], which would imply that very heavy neutrinos, with masses larger than $10^{13} \mathrm{GeV}$ would not be significantly produced in the reheating. Even stronger constraints on $T_{R H}$ have been obtained by considering non thermal production of gravitinos [4.

More recently, the neutrino production has been also analyzed in the so-called preheating scenario [5], which corresponds to a resonant particle production during the first inflaton oscillations around the minimum of the potential. The main result of this mechanism is that even neutrinos with masses of the order of $10^{15} \mathrm{GeV}$ can be efficiently produced in highly non-thermal momentum distribution. As will be clear in the follow- 
ing, this feature of $N_{i}$ energy spectrum distribution is crucial for our purposes.

The second issue is related to the presence of $C P$ violating contributions to the neutrino decay channels in massless fermions and Higgs bosons, and to the role of interference effects which make this $C P$ violation observable. There are two such contributions to the microscopic asymmetry $\epsilon$ which have been considered in literature. The first one identified [1] is due to interference of the tree-level amplitudes with the absorptive part of the one loop vertex $\left(\epsilon^{\prime}\right.$-like effect)

$$
\epsilon_{v}=-\frac{1}{8 \pi}\left[\left(h^{\dagger} h\right)_{11}\right]^{-1} \sum_{j} \mathcal{I} m\left[\left(h^{\dagger} h\right)_{1 j}^{2}\right] f\left(\frac{m_{j}^{2}}{m_{1}^{2}}\right)
$$

with $m_{1}$ the mass of the lightest Majorana neutrino, $f(x)$ can be found in Ref. [1] and $h$ is the coupling of $N_{i}$ to massless left-handed leptons and Higgs bosons.

Recently, it has been observed that one should also consider contributions coming via interference with one loop self-energy ( $\epsilon$-like effect) [6]-[9]. For example for each neutrino $N_{i}$ one has

$$
\epsilon_{s}^{i}=-\frac{1}{8 \pi} \sum_{j \neq i} \frac{m_{i} m_{j}}{m_{i}^{2}-m_{j}^{2}} \frac{\mathcal{I} m\left[\left(h^{\dagger} h\right)_{i j}^{2}\right]}{\left(h^{\dagger} h\right)_{i i}} .
$$

It is worth observing that both asymmetries (2) and (3) very much resemble the $C P$ violation asymmetries for charged $B$ mesons

$$
\epsilon_{f}=\frac{\Gamma\left(B^{+} \rightarrow f\right)-\Gamma\left(B^{-} \rightarrow f^{c}\right)}{\Gamma\left(B^{+} \rightarrow f\right)+\Gamma\left(B^{-} \rightarrow f^{c}\right)}
$$

with $f$ denoting an arbitrary final state. As for $\epsilon_{v}$ and $\epsilon_{s}$, in order to have a not vanishing result one needs more than one contribution to the exclusive decay channel $f$. In complete analogy to lepton asymmetries, the additional contribution to exclusive $B^{ \pm}$decay channels is provided by radiative processes, the so-called penguin diagrams.

In this paper we consider a different scenario for neutrino decays, which provides already at tree-level in the amplitudes a microscopic contribution to the lepton asymmetry. The mechanism, following again the analogy with $B$ physics, is much reminding the time integrated $C P$ asymmetry in the $B^{0}-\bar{B}^{0}$ system [10]. In this case, differently from the charged $B$, by virtue of $B^{0}-\bar{B}^{0}$ oscillations one can produce $C P$ asymmetries already at tree-level. 
We consider the case in which heavy Majorana neutrinos are produced out of thermal equilibrium through a preheating mechanism. For arbitrary couplings of these neutrinos to the inflaton field, they are produced as superpositions of the mass eigenstates $N_{i}$, that we denote as inflaton eigenstates, $N_{\alpha}$. If neutrino lifetimes are less than the typical decoherence time due to scattering processes in the medium, these states, once produced, propagate as coherent superpositions of the $N_{i}$ till their eventual decay, if neutrino lifetimes are less than the typical decoherence time due to scatterings in the medium. This constraint leads to conditions on both the couplings $h$ and the Majorana masses which, however, are neither particularly severe nor fine tuned.

As in the usual scenario, lepton number is produced via decays in (massless) lefthanded fermions $\psi_{L j}$ ( $j$ being the family index) and Higgs bosons $\Phi$, and their $C$-conjugate particles, right-handed antifermions $\psi_{R j}^{c}$ and $\Phi^{c}$, giving rise to a microscopic asymmetry, for each $\alpha$

$$
\epsilon_{\alpha}=\sum_{j=1}^{n_{g}} \frac{\Gamma\left(N_{\alpha} \rightarrow \psi_{L j} \Phi\right)-\Gamma\left(N_{\alpha} \rightarrow \psi_{R j}^{c} \Phi^{c}\right)}{\Gamma\left(N_{\alpha} \rightarrow \psi_{L j} \Phi\right)+\Gamma\left(N_{\alpha} \rightarrow \psi_{R j}^{c} \Phi^{c}\right)}
$$

Since each $N_{\alpha}$ is a quantum superposition of the mass eigenstates $N_{i}$, their decay amplitudes are linear combinations of $\mathcal{A}\left(N_{i} \rightarrow \psi_{L j} \Phi, \psi_{R j}^{c} \Phi^{c}\right)$. Provided the Yukawa matrix explicitly breaks $C P$ invariance, a not vanishing value for $\epsilon_{\alpha}$ can be obtained at tree-level only if these different amplitudes may interfere.

It should be stressed at this point that it is always difficult to deal with unstable particles in the clean framework of Quantum Field Theory, since, in this case, they cannot be identified with asymptotic states in some Hilbert space, but rather they appear as resonances in S-matrix elements. An evaluation of the asymmetries $\epsilon_{v}$ and $\epsilon_{s}$ in this approach has been done in [11]. In our case, one should consider the decay of the inflaton field into the states $\psi_{L j} \Phi, \psi_{R j}^{c} \Phi^{c}$ which resonantly proceeds via intermediate $N_{\alpha}$. If, however, the heavy neutrinos have very small decay widths compared with their masses, which we will always assume in the following, one may envisage this process as the production of the (quasi)-stable state $N_{\alpha}$ and its subsequent decay. To describe this process we will introduce a neutrino wave function, accounting for its exponential decay, whose espression can be deduced from the form of the corresponding propagator in the vicinity of the resonance. 
The basic idea of the paper is as follows. Let us consider an arbitrary final state, like $\psi_{L j} \Phi$ or $\psi_{R j}^{c} \Phi^{c}$, with invariant mass $\mu$. It can be produced by the decay of neutrinos with mass $m_{i}$ if $\mu$ is, say, in the range $m_{i}-\Gamma_{i} / 2 \leq \mu \leq m_{i}+\Gamma_{i} / 2$. If there are two such neutrinos $N_{i}$ and $N_{k}$, whose mass difference is smaller than their average width $\Gamma_{i k}=\left(\Gamma_{i}+\Gamma_{k}\right) / 2$, it is impossible to distinguish if this decay product is the result of the decay of $N_{i}$ or rather $N_{k}$. In other words, for the process $N_{\alpha} \rightarrow \psi_{L j} \Phi$ one expects in this case the contribution of the amplitudes due to both possible processes: $N_{\alpha} \rightarrow N_{i} \rightarrow \psi_{L j} \Phi$ and $N_{\alpha} \rightarrow N_{k} \rightarrow \psi_{L j} \Phi$.

The interference between the two amplitudes leads, in general, to not vanishing microscopic asymmetries $\epsilon_{\alpha}$, which are found to be proportional to the factors $\left(m_{i}-m_{j}\right) / \Gamma_{i j}$ and to the imaginary parts of the matrix products $h h^{\dagger}$. Of course, if the Majorana neutrinos would be absolutely stable states, or, in the case of decaying particles, if their mass differences are much greater than their widths $\Gamma_{i}$, any interference would be impossible and the resulting asymmetry would vanish.

The paper is organized as follows. In section 2 we briefly summarize the main features of the model describing heavy Majorana neutrino dynamics, whose out of equilibrium production mechanism is discussed in section 3. The estimate of the resulting macroscopic $L$ asymmetry is presented in section 4 . Finally, section 5 contains our conclusions and outlooks.

\section{The model}

Let us consider heavy neutrinos $\nu_{R i}$ and $\nu_{L i}^{c}(i$ denotes the family index) with a Majorana mass term

$$
L_{M}=-\left(\bar{\nu}_{L i}^{c} M_{i j} \nu_{R j}+\bar{\nu}_{R i} M_{i j} \nu_{L j}^{c}\right)
$$

with $M$ a symmetric real matrix. This mass term can be diagonalized in terms of a set of Majorana neutrinos $N_{i}$, with a latin letter as family index, defined as follows

$$
\nu_{R i}=P_{R} W_{i j} N_{j} \quad, \quad \nu_{L i}^{c}=P_{L} W_{i j} N_{j}
$$

where $W$ is an orthogonal matrix such that $W^{T} M W$ is diagonal, and $P_{R, L} \equiv\left(1 \pm \gamma_{5}\right) / 2$. Denoting with $\chi$ the scalar field behaving, in a certain period of the evolution of the uni- 
verse, as the inflaton, the production of the heavy neutrinos via the reheating mechanism takes place due to a Yukawa term in the Lagrangian density of the form

$$
L_{\chi}=-\chi\left(\bar{\nu}_{L i}^{c} G_{i j} \nu_{R j}+\bar{\nu}_{R i} G_{i j} \nu_{L j}^{c}\right)
$$

where again $G$ is a symmetric real matrix. Let us denote with $N_{\alpha}$ (with a greek letter as index) the basis of $G$ eigenstates. In this basis the Majorana mass matrix $M$ is in general not diagonal, the two basis being connected by an orthogonal transformation

$$
N_{\alpha}=U_{\alpha i} N_{i}
$$

Finally, the heavy neutrinos are also coupled to massless left-handed leptons and to the standard $S U(2)$ Higgs doublet $\Phi$ through a Dirac term

$$
L_{D}=-\left(\bar{\psi}_{R i}^{c} \cdot \Phi\right) h_{i j}^{*} N_{j}-\left(\bar{\psi}_{L i} \cdot \Phi^{c}\right) h_{i j} N_{j}
$$

with $h$ the Yukawa coupling matrix in the family space and

$$
\psi_{L i}=\left(\begin{array}{c}
\nu_{L i} \\
l_{L i}^{-}
\end{array}\right) \quad, \quad \Phi=\left(\begin{array}{c}
\varphi^{+} \\
\varphi^{0}
\end{array}\right) .
$$

\section{Neutrino production}

In the usual scenario, neutrinos, along with all species of particles with masses below the maximal temperature achieved during reheating, are produced as thermal excitations when the inflaton releases its energy density and the radiation epoch starts. This means that, if the $N_{i}$ have masses smaller than this temperature, they can be thermally excited. In this case, the $N_{i}$ field configuration would correspond to a thermal distribution of particles with definite mass given by the eigenvalues $m_{i}$ of the Majorana mass matrix $M$. When eventually the temperature decreases down to $m_{i}$, if the decays into massless leptons and Higgses take place in a out-of-equilibrium condition, then a macroscopic lepton asymmetry can be produced, provided all Sacharov conditions are satisfied. This scenario has been widely studied, and, as mentioned in the previous section, the effect is basically due to the interference between the tree-level decay amplitudes and the one-loop contributions [1, 6]. In light of the bounds on $T_{R H}$ and the discussion in the Introduction, this scenario seems only viable for Majorana neutrinos with masses lighter than $10^{13} \mathrm{GeV}$. 
We would rather analyze the case when these neutrinos are produced through a preheating mechanism as coherent superpositions of mass eigenstates. In this scenario particles are resonantly excited due to the oscillatory behaviour of the inflaton field $\chi(t)=\chi_{0} \cos \left(m_{\chi} t\right), m_{\chi}^{2}$ being the second derivative of the inflaton potential at its minimum. In the following we will consider the simplest case of a quadratic potential for $\chi$. It has been shown by several authors, [4], [5], [12]- [16], that is possible to produce spin(0), spin(1/2), as well as spin(3/2) particles through non-perturbative effects. The neutrino field satisfies a Dirac equation on a Friedmann-Robertson-Walker spacetime with an effective time dependent mass matrix $\mathcal{M}$. In conformal time $d \eta=d t / R(t)$, with $R(t)$ the scale factor

$$
\left[\frac{i}{R} \gamma^{\mu} \partial_{\mu}+i \frac{3}{2 R^{2}} \frac{d R}{d \eta} \gamma^{0}\right] N_{i}=\mathcal{M}_{i j}(\eta) N_{j}
$$

with

$$
\mathcal{M}_{i j}(\eta)=M_{i j}+\chi(\eta) G_{i j}
$$

Equation (12) describes oscillators with a complex time varying frequency. In the simple case of diagonal $M$ and $G$ matrices the neutrino number density is obtained by using a time dependent Bogoliubov canonical transformation [5]. In the general case, however, since $M$ and $G$ are not necessarily simultaneously diagonal one should first diagonalize via an orthogonal transformation the effective mass $\mathcal{M}$, whose eigenvalues may have quite an involved dependence on $\chi(\eta)$. Let us consider the two possible cases:

i) if the order of magnitude of the matrix elements of $M$ is much larger than the one of $\chi(\eta) G$, we may treat the term $\chi(\eta) G$ as a perturbation to $M$. Thus in the Majorana mass eigenbasis, up to the first order in perturbation theory we get

$$
\mathcal{M}_{i j}(\eta) \simeq\left[m_{i}+\chi(\eta) G_{i i}\right] \delta_{i j}
$$

Hence the $n_{g}$ equations for $i=1,2, . ., n_{g}$ are decoupled and can be treated as in Ref. [5]. In this case the inflaton would produce neutrinos already as mass eigenstates, but the resonant condition $\operatorname{det}[\mathcal{M}(\eta)]=0$ for an explosive production of heavy neutrinos is never satisfied.

ii) in the opposite case, when the time dependent mass term, provided by the coupling to the inflaton, is comparable or even dominant over $M$, it is convenient to use the 
inflaton basis to write the mass term as

$$
\mathcal{M}_{\alpha \beta}(\eta) \simeq\left[M_{\alpha \beta}+\chi(\eta) g_{\alpha} \delta_{\alpha \beta}\right]
$$

Thus, the preheating neutrino production occurs for those values of $\eta$ for which $\operatorname{det}[\mathcal{M}(\eta)]$ vanishes.

In case $i i$ ), if we assume, as it will be clear in the following, small off-diagonal terms in the matrix $M$ compared to the diagonal ones, we can write the preheating production condition as

$$
\operatorname{det}[\mathcal{M}(\eta)]=\Pi_{\alpha=1}^{n_{g}}\left(M_{\alpha \alpha}+\chi(\eta) g_{\alpha}\right)+O\left(\frac{m_{\alpha \beta}}{\bar{m}}\right)=0
$$

where $m_{\alpha \beta}$ just denotes the order of magnitude of the off-diagonal mass terms in $M$ and $\bar{m}$ the one of the diagonal entries. At lowest order in the ratio $m_{\alpha \beta} / \bar{m}$, eq. (16) is satisfied if, for some $\eta_{*}$

$$
M_{\alpha \alpha}+\chi\left(\eta_{*}\right) g_{\alpha}=0
$$

In this case $N_{\alpha}$ heavy Majorana neutrinos will be resonantly produced. However, since the conditions (16) is only satisfied up to terms of the order $m_{\alpha \beta} / \bar{m}$, this implies that the production rates are suppressed to some extent. In other words it is as these neutrinos at $\eta_{*}$ would not be produced as massless but with a mass of the order of $m_{\alpha \beta}^{2} / \bar{m}$. This effect which leads to an exponential suppression factor of the number of heavy Majorana neutrinos produced [5], can be neglected if the following condition is satisfied

$$
\frac{m_{\alpha \beta}^{2}}{\bar{m}}<<\sqrt{g_{\alpha} \chi^{\prime}\left(\eta_{*}\right)}
$$

Since $g_{\alpha} \chi^{\prime}\left(\eta_{*}\right)=g_{\alpha} m_{\chi} \chi\left(\eta_{*}\right) \sim \bar{m} m_{\chi}$, the above condition becomes

$$
\frac{m_{\alpha \beta}}{\bar{m}}<<\left(\frac{m_{\chi}}{\bar{m}}\right)^{1 / 4}
$$

The constraint (19) gives for example for the typical values $m_{\chi}=10^{13} \mathrm{GeV}$ and $\bar{m}=$ $10^{15} \mathrm{GeV}, m_{\alpha \beta} / \bar{m} \leq 10^{-1}$, which does not severely affects the order of magnitude of the off-diagonal elements in $M_{\alpha \beta}$, and still allows for a quite large mixing, given by the non diagonal elements of the matrix $U$, see eq. (9).

Under the condition (19) one can safely apply the results of Ref. [5] where to solve eq. (12), in case $i i$ ), one writes down the momentum expansion for Majorana neutrinos 
$N_{\alpha}(\eta, \vec{x})$. By a time dependent Bogoliubov transformation it is then possible to diagonalize the Hamiltonian in terms of quasi-particle creation and annihilation operators and, with a customary procedure, to deduce the number of produced particles as the expectation value of the particle number operator $n_{\alpha}$ on the quasi-particle vacuum. Depending on the value of the parameter $q_{\alpha}$, defined as

$$
q_{\alpha} \equiv \frac{g_{\alpha}^{2} \chi^{2}(0)}{4 m_{\chi}^{2}}
$$

with $\chi(0)$ the initial value of the inflaton field configuration, the final number density quite rapidly reaches the bound due to Pauli blocking and can be expressed in terms of the maximal momentum $k_{\max }$ of the distribution

$$
n_{\alpha} \simeq k_{\max }^{3} \simeq m_{\chi}^{2} M_{\alpha \alpha}
$$

At later times, when eventually oscillations are damped to smaller values, the fraction of the inflaton energy density transferred to heavy neutrinos, $\rho_{\alpha} / \rho_{\chi}$, is frozen to the value, see Ref. [5],

$$
\frac{\rho_{\alpha}}{\rho_{\chi}} \simeq \frac{m_{\chi}^{2}}{\chi^{2}(0)} q_{\alpha} .
$$

In the framework of a chaotic inflation scenario, from the observed amplitude of the density perturbations on large scales, $m_{\chi}$ is constrained to be of the order of $10^{13} \mathrm{GeV}$. Furthermore, with $\chi(0) \simeq m_{P l}$, one gets $\rho_{\alpha} / \rho_{\chi} \simeq 10^{-12} q_{\alpha}$. In the following this result will be used to estimate the final lepton number produced by heavy neutrinos.

\section{Neutrino propagation and decay}

In the previous section we have described the production mechanism of the $N_{\alpha}$ neutrinos regarded as asymptotic stable states. In principle the neutrino production by the inflaton, and their subsequent decays in fermion-Higgs pairs should be considered as a whole, $\chi \rightarrow$ $N_{\alpha} \rightarrow \psi_{L j} \Phi, \psi_{R j}^{c} \Phi^{c}$. The metastable character of the $N_{\alpha}$ would therefore be encoded in the expression of its propagator. However it should be first noted that the nonperturbative mechanism outlined in the previous section is basically istantaneous [5]. If in addition the $N_{\alpha}$ are sufficiently long-lived, i.e. the widths are small compared with their masses, 
we can safely describe the process as occurring in three stages: the production of the quasi-stable states $N_{\alpha}$, its propagation in the medium and its eventual decay.

If neutrinos are produced in the inflaton basis $N_{\alpha}$, say at time $t=0$ and with momentum $\vec{k}$, they start evolving as a linear superposition of the mass eigenstates $N_{j}$ of eigenvalue $m_{j}$ as follows

$$
N_{\alpha}(x ; \vec{k})=U_{\alpha j} N_{j}(x ; \vec{k})
$$

with $x \equiv(t, \vec{x})$ and $N_{j}(x ; \vec{k})$ the wave-function of the mass eigenstate $N_{j}$. The form of this wavefunction, which account for the metastable behaviour of $N_{j}$ and can be inferred from the form of the propagator, will be discussed in the following. In presence of a medium, scattering processes will tend to destroy the coherence among the components of the wave function $N_{\alpha}(x ; \vec{k})$ before its decay, and to populate the universe with thermalized neutrino mass eigenstates. In order to avoid this, one has to impose the condition

$$
\Gamma_{\alpha}>>n\langle\sigma\rangle \equiv \Gamma_{s c}
$$

where $\Gamma_{\alpha}$ denotes the $N_{\alpha}$ decay rate

$$
\Gamma_{\alpha}=U_{\alpha i}\left(\sum_{f}\left\langle f \mid N_{i}\right\rangle\left\langle N_{j} \mid f\right\rangle\right) U_{j \alpha}^{T}
$$

where the sum is over all possible final states $f$. With $\sigma$ we denote the cross section of the relevant scattering processes, averaged over the incoming particles distribution with number density $n$. The dominant contribution to $\Gamma_{\alpha}$ comes from the two body decay channels $\psi_{L i} \Phi$. If the neutrino masses are of the same order of magnitude $m_{i} \simeq \bar{m} \forall i$, the factor in bracket in eq. (25) simplifies to

$$
\sum_{f}\left\langle f \mid N_{i}\right\rangle\left\langle N_{j} \mid f\right\rangle \simeq\left(h h^{\dagger}\right)_{i j} \frac{\bar{m}}{8 \pi} \Rightarrow \Gamma_{\alpha} \simeq\left(h h^{\dagger}\right)_{\alpha \alpha} \frac{\bar{m}}{8 \pi}
$$

Notice that the case of almost mass degenerate neutrinos is actually the scenario we are mostly interested in this paper.

The main contributions to $\Gamma_{s c}$ correspond to the processes $N_{\alpha} \Phi \longrightarrow N_{i} \Phi, N_{\alpha} \psi_{L i} \longrightarrow$ $N_{i} \psi_{L j}$ and crossed channels, as well as to scattering over the large amount of inflaton quanta $\chi$. Condition (25) constraints more severely the Yukawa couplings $h$ and $G$ when the mean energy of the massless $\Phi, \psi_{L i}$ and $\chi$ is larger than the neutrino masses. This is 
due to the rapid increasing with this mean energy of the product $n_{\Phi, \psi_{L}, \chi}\langle\sigma\rangle$. In fact, defining, in the $N_{\alpha}$ rest frame, the effective temperature $T_{*}$, which represents the mean energy of the massless fermions or Higgses, and similarly $T_{\chi}$ the one of the inflaton excitations, we have, for scattering over massless fermions and Higgses

$$
\left\langle\sigma\left(\psi_{L}, \Phi\right)\right\rangle \simeq \frac{1}{8 \pi \bar{m} T_{*}}\left[\left(h h^{\dagger}\right)_{\alpha \alpha} \sum_{j=1}^{n_{g}}\left(h h^{\dagger}\right)_{j j}+\left(h h^{\dagger} h h^{\dagger}\right)_{\alpha \alpha}\right],
$$

and similarly, for scattering processes over $\chi$ bosons

$$
\langle\sigma(\chi)\rangle \sim \frac{1}{8 \pi \bar{m} T_{\chi}}\left(G^{4}\right)_{\alpha \alpha} .
$$

For low momentum neutrinos $T_{*}$ and $T_{\chi}$ also give the mean energy in the comoving frame. Actually from the discussion of section 3 , we see that in our preferred choice for $\bar{m} \geq 10^{13}$ $\mathrm{GeV}$ and $m_{\chi} \sim 10^{13} \mathrm{GeV}$, neutrinos are basically emitted as non-relativistic particles, since $k_{\max } / \bar{m} \simeq\left(m_{\chi} / \bar{m}\right)^{2 / 3} \leq 1$, thus we can safely neglect any effect due to the difference between the neutrino rest frame and the comoving frame. Since the number density of incoming particles can be expressed as $n_{\psi_{L}} \simeq n_{\Phi} \simeq T_{*}^{3}, n_{\chi} \simeq T_{\chi}^{3}$, the condition for no decoherence becomes

$$
\frac{\left(h h^{\dagger}\right)_{\alpha \alpha} \sum_{j=1}^{n_{g}}\left(h h^{\dagger}\right)_{j j}+\left(h h^{\dagger} h h^{\dagger}\right)_{\alpha \alpha}}{\left(h h^{\dagger}\right)_{\alpha \alpha}} T_{*}^{2}+\frac{\left(G^{4}\right)_{\alpha \alpha}}{\left(h h^{\dagger}\right)_{\alpha \alpha}} T_{\chi}^{2} \leq \bar{m}^{2} .
$$

No particularly fine tuned condition on the Yukawa couplings $h$ and $G$ follows from (29) even for very heavy Majorana neutrinos, $\bar{m} \sim 10^{15} \mathrm{GeV}$. Assuming in fact the extreme limit $T_{\chi} \sim\left(10^{-2} \div 10^{-1}\right) m_{P l}$ which, still compatible with a classical description of spacetime structure, seems to be suggested by an exponential production of $\chi$ quanta by the inflaton oscillating configuration [17], we get, as an extremely conservative estimate, that there is no decoherence if we take the Yukawa couplings $G, h$ up to $\left(10^{-3} \div 10^{-2}\right)$. This implies the conservative upper bound for $q_{\alpha}(20)$

$$
q_{\alpha} \lesssim\left(10^{6} \div 10^{8}\right)
$$

If condition (29) is satisfied, heavy neutrino states (23) evolve coherently till their decays into massless fermions and Higgses.

To evaluate the decay rates in these channels we first have to specify the expression for the wave functions $N_{j}(x ; \vec{k})$. For this purpose we first recall that, in a neighborhood of the 
mass eigenvalues $m_{j}$, the $N_{j}$ propagator $G_{j}\left(p^{2}\right)$ gets the familiar Breit-Wigner behaviour. Using Lehman spectral decomposition we can also write $G_{j}\left(p^{2}\right)$ as the superposition of free propagators of given masses via two spectral functions

$$
G_{j}\left(p^{2}\right)=\frac{\not p+m_{j}}{p^{2}-m_{j}^{2}+i m_{j} \Gamma_{j}} \mathcal{C}^{-1}=\int_{0}^{\infty} d \mu^{2}\left(\not p w_{j}^{1}\left(\mu^{2}\right)+w_{j}^{2}\left(\mu^{2}\right)\right) \frac{1}{p^{2}-\mu^{2}+i \epsilon} \mathcal{C}^{-1}
$$

with $\mathcal{C}$ the charge conjugation operator. For $\mu^{2} \sim m_{j}^{2}$, it is straightforward to get

$$
w_{j}^{1}\left(\mu^{2}\right)=\frac{w_{j}^{2}\left(\mu^{2}\right)}{m_{j}}=\frac{m_{j} \Gamma_{j}}{\pi\left[\left(\mu^{2}-m_{j}^{2}\right)^{2}+m_{j}^{2} \Gamma_{j}^{2}\right]} .
$$

This result follows by expanding the free propagators as the principal value of $\left(p^{2}-\mu^{2}\right)^{-1}$ and its Dirac $\delta$-contribution.

The positive definite spectral function $w_{j}^{1}$ can be simply related to the wave function $\rho_{j}$ of the unstable state $N_{j}$ in configuration space. If we consider the expansion of the field $\mathcal{N}_{j}$, which represents the Majorana neutrinos, as follows

$$
\mathcal{N}_{j}(x)=\int \frac{d^{3} k}{2 k^{0}}\left[N_{j}(x ; \vec{k}) a_{\vec{k}}+N_{j}^{c}(x ; \vec{k}) a_{\vec{k}}^{\dagger}\right]
$$

with $N_{j}(x ; \vec{k})$ a set of neutrino wavefunction with spatial momentum $\vec{k}$, encoding its unstable character, it is straightforward to see that we recover the correct Breit-Wigner behaviour of the corresponding propagator if we represent these wavefunctions as

$$
N_{j}(x ; \vec{k})=\int d \mu^{2} \rho_{j}\left(\mu^{2}\right) u_{\vec{k}}^{\mu} e^{-i k \cdot x}
$$

with $\left|\rho_{j}\left(\mu^{2}\right)\right|^{2}=w_{j}^{1}\left(\mu^{2}\right)$. We have denoted with $u_{\vec{k}}^{\mu}(x)$ a four component spinor solution of the Dirac equation in momentum space with mass $\mu$ and momentum $\vec{k}$. The spectral function $w_{j}^{1}$ does not uniquely fix the wavefunctions $N_{j}(x ; \vec{k})$. The simplest choice for $\rho_{j}\left(\mu^{2}\right)$, satisfying $\left|\rho_{j}\left(\mu^{2}\right)\right|^{2}=w_{j}^{1}\left(\mu^{2}\right)$, is again a Breit-Wigner function

$$
\rho_{j}\left(\mu^{2}\right)=\frac{\sqrt{m_{j} \Gamma_{j}}}{\sqrt{\pi}\left(\mu^{2}-m_{j}^{2}+i m_{j} \Gamma_{j}\right)} .
$$

We stress that in what follows it is not really crucial the particular choice for $\rho_{j}\left(\mu^{2}\right)$, satisfying $\left|\rho_{j}\right|^{2}=w_{j}^{1}$. The only relevant aspect we have to require in order to get a non vanishing lepton asymmetry is that $\rho_{j}\left(\mu^{2}\right)$ has an imaginary part depending on the two parameters $m_{j}$ and $\Gamma_{j}$. This assumption is quite robust, if we one recall the fact that phase 
shifts due to the production of any resonance is strongly dependent on these quantities. Finally we observe that in the limit of vanishing decay widths $\rho_{j}\left(\mu^{2}\right) \rightarrow \delta\left(\mu^{2}-m_{j}^{2}\right)$, so in this limit $N_{j}(x ; \vec{k}) \rightarrow u_{\vec{k}}^{m_{j}}(x) e^{-i k \cdot x}$, which describes a stable neutrino with mass $m_{j}$ and momentum $\vec{k}$

We can now evaluate the decay rates of the $N_{\alpha}$. As we have discussed in the preheating mechanism neutrinos are basically produced with low momenta, much smaller than their masses. It is therefore a good approximation to consider the case of neutrinos emitted at rest in the comoving frame. From eq. (21) all corrections to the results below are at most of the order of $\left(\bar{m} / m_{\chi}\right)^{2 / 3}$.

Depending on the Majorana neutrino mass spectrum, it is possible that two or more $\rho_{j}$ significantly overlap. This occurs whenever $\left|m_{i}-m_{j}\right|<\left(\Gamma_{i}+\Gamma_{j}\right) / 2$. Using (34), the total decay rate of $N_{\alpha}$ into pairs $\psi_{L p} \Phi$ as well as into the $C$-conjugated channels $\psi_{R p}^{c} \Phi^{c}$ is given by

$$
\sum_{p=1}^{n_{g}} \Gamma\left(N_{\alpha} \rightarrow \psi_{L p} \Phi\right)=\Xi_{\alpha}^{i j} I_{i j} \quad, \quad \sum_{p=1}^{n_{g}} \Gamma\left(N_{\alpha} \rightarrow \psi_{R p}^{c} \Phi^{c}\right)=\left(\Xi_{\alpha}^{i j}\right)^{*} I_{i j}
$$

where

$$
\begin{aligned}
\Xi_{\alpha}^{i j} & =U_{\alpha i}\left(h h^{\dagger}\right)_{i j} U_{\alpha j}=\left(\Xi_{\alpha}^{j i}\right)^{*}, \text { no sum over } i \text { and } j, \\
I_{i j} & =\frac{1}{8 \pi} \int_{0}^{\infty} \frac{\mu^{2} d \mu}{\sqrt{|\vec{k}|^{2}+\mu^{2}}} \rho_{i}(\mu) \rho_{j}^{*}(\mu) \sim \frac{1}{8 \pi} \int_{0}^{\infty} \sqrt{\mu^{2}} d \mu \rho_{i}(\mu) \rho_{j}^{*}(\mu)=I_{j i}^{*}
\end{aligned}
$$

In eq. (38) the factor $\mu^{2} /\left(8 \pi \sqrt{|\vec{k}|^{2}+\mu^{2}}\right) \sim \mu /(8 \pi)$ is the result of integration over the phase space for final massless particles with fixed initial mass $\mu$ and momentum $\vec{k}$. Notice also that we have integrated in $I_{i j}$ over the range $[0, \infty[$, rather than in the narrow neighborhood of the masses $m_{i}$ and $m_{j}$. This is justified by observing that $I_{i j}$ receive the main contribution from these neighborhoods, since the products $\rho_{i}\left(\mu^{2}\right) \rho_{j}^{*}\left(\mu^{2}\right)$ are rapidly decreasing functions for $\mu^{2}<<m_{i}^{2}, m_{j}^{2}$ and $\mu^{2}>>m_{i}^{2}, m_{j}^{2}$.

A straightforward computation shows that the microscopic asymmetries $\epsilon_{\alpha}$ are given by

$$
\epsilon_{\alpha}=\frac{2 \sum_{i<j} \mathcal{I} m\left[\Xi_{\alpha}^{i j}\right] \mathcal{I} m\left[I_{i j}\right]}{2 \sum_{i<j} \mathcal{R} e\left[\Xi_{\alpha}^{i j}\right] \mathcal{R} e\left[I_{j i}\right]+\sum_{i=1}^{n_{g}} \Xi_{\alpha}^{i i} I_{i i}}
$$

From this result we get that, in order to have at tree-level not vanishing microscopic asymmetries it is necessary that: 
i) a $C P$ violating term is contained in the Yukawa couplings $h$;

ii) the integrals $I_{i j}$ of the spectral functions contain a not vanishing imaginary part.

As already stated, this second condition is realized if at least two of the neutrino masses satisfy the condition $\left|m_{j}-m_{i}\right| \leq\left(\Gamma_{j}+\Gamma_{i}\right) / 2$, otherwise the two kernels $\rho_{i}\left(\mu^{2}\right) \rho_{j}\left(\mu^{2}\right)$ have no significant overlap. If $m_{j}>m_{i}$, the main contribution to the imaginary part of $I_{i j}$ is expected for $m_{i}<\mu<m_{j}$. In this interval the phase difference of $\rho_{i}$ and $\rho_{j}$ is almost $\pi$. Furthermore, even if $m_{i}=m_{j}$, an imaginary part for $I_{i j}$ is expected if the two widths are sensibly different.

Defining $\Gamma_{i j} \equiv\left(\Gamma_{i}+\Gamma_{j}\right) / 2, m_{i j} \equiv\left(m_{i}+m_{j}\right) / 2, \Delta_{i j} \equiv\left(m_{j}-m_{i}\right) /\left(\Gamma_{i}+\Gamma_{j}\right)$ and $\gamma_{i j} \equiv\left(\Gamma_{j}-\Gamma_{i}\right) / \Gamma_{i j}$, and if we also assume for simplicity that both $\Delta_{i j}, \gamma_{i j}<1$, the asymmetry can be obtained as an expansion in powers of these parameters. In the narrow width limit, a simple calculation up to the first order in $\Delta_{i j}$ and $\gamma_{i j}$ gives

$$
I_{i j} \simeq \frac{1}{8 \Gamma_{i j}}\left(1+i \Delta_{i j}+i \gamma_{i j} \frac{\Gamma_{i j}}{m_{i j}}\right)
$$

It is interesting to consider the case of only two generations, for which the expression of the $L$ microscopic asymmetry is particularly simple and the orthogonal matrix $U$ is given by

$$
U=\left(\begin{array}{cc}
\cos (\theta) & \sin (\theta) \\
-\sin (\theta) & \cos (\theta)
\end{array}\right)
$$

A simple calculation gives at the lowest order in $\Delta_{12}$ and $\gamma_{12}$

$$
\begin{aligned}
\epsilon_{1,2} & = \pm \frac{2 \sin (2 \theta) \mathcal{I} m\left[\left(h h^{\dagger}\right)_{12}\right]}{\left(h h^{\dagger}\right)_{11}+\left(h h^{\dagger}\right)_{22} \mp \sin (2 \theta) \mathcal{R} e\left[\left(h h^{\dagger}\right)_{12}\right]}\left(\Delta_{12}+\gamma_{12} \frac{\Gamma_{12}}{m_{12}}\right) \\
& = \pm \sin (2 \theta) \lambda_{C P}\left(\Delta_{12}+\gamma_{12} \frac{\Gamma_{12}}{m_{12}}\right) \frac{\left(h h^{\dagger}\right)_{11}+\left(h h^{\dagger}\right)_{22}}{\left(h h^{\dagger}\right)_{11}+\left(h h^{\dagger}\right)_{22} \mp \sin (2 \theta) \mathcal{R} e\left[\left(h h^{\dagger}\right)_{12}\right]}
\end{aligned}
$$

with

$$
\lambda_{C P}=\frac{2 \mathcal{I} m\left[\left(h h^{\dagger}\right)_{12}\right]}{\left(h h^{\dagger}\right)_{11}+\left(h h^{\dagger}\right)_{22}}
$$

representing the strength of the $C P$ violating effects. Notice that since we are working with Majorana neutrinos, even for two generations a phase in the matrix $h$ cannot be washed away by a simple redefinition of fields, so $\left(h h^{\dagger}\right)_{12}$ is in general a complex quantity.

There are several features of eq. (42) which is worth observing. First of all the two asymmetries tend to cancel each other, simply because the two numerators of $\epsilon_{1}$ and $\epsilon_{2}$ 
are opposite by the orthogonality of the mixing matrix $U$. This is exactly analogous to GIM mechanism [18]. However, already at first order the sum $\epsilon_{1}+\epsilon_{2}$ does not vanish because the denominator in eq. (42) is different for the two neutrinos. Furthermore, the total lepton asymmetry $L$ is given by the microscopic asymmetries weighted by the corresponding neutrino number densities $n_{i}$. As long as $n_{1} \neq n_{2}$ the value of $L$ is not expected to vanish, though a partial cancellation still takes place. It should also be pointed out that at lower order there is also a contribution from the widths difference parameter $\gamma_{12}$, which is however suppressed by the small factor $\Gamma_{12} / m_{12}$.

Finally notice that the value for the asymmetry is obtained at tree-level in decay amplitudes, while both contributions previously considered, $\epsilon_{v}$ and $\epsilon_{s}$, depend on matrix elements of $\left(h h^{\dagger}\right)^{2}$ in the numerator, see eq.s (2),(3). This means that the asymmetries (42) can be in principle quite large since they are not suppressed by higher powers of the Yukawa couplings, though there is a certain cancellation among them. Also notice that, differently than eq. (3), $\epsilon_{1,2}$ are only linearly dependent on $\Delta_{12}$, so they are less suppressed in the limit of small $\Delta_{12}$. Incidentally, in the usual scheme the Yukawa matrix elements are constrained to be quite small $\left(h h^{\dagger}\right)_{i i} \leq m_{i} / m_{P l}$ to have an out-of-equilibrium decay. This bound is no more necessary if the neutrino are already produced, as in the preheating scenario, in a non thermal way, and the reheating temperature $T_{R H}$ is low enough to prevent from a subsequent thermal production of $N_{i}$.

Using the results of section 3 , we can finally estimate the total lepton number $n_{L}$, normalized to specific entropy. Since the energy fractions $\rho_{\alpha}$ remain constant till the inflaton decay into radiation, after the reheating stage one gets

$$
\frac{n_{L}}{s} \simeq \frac{T_{R H}}{\bar{m} \rho_{\chi}} \sum_{\alpha=1}^{n_{g}} \epsilon_{\alpha} \rho_{\alpha} \simeq 10^{-17}\left(\frac{T_{R H}}{10^{10} G e V}\right)\left(\frac{10^{15} G e V}{\bar{m}}\right) \sum_{\alpha=1}^{n_{g}} \epsilon_{\alpha} q_{\alpha} .
$$

Choosing $q_{\alpha}$ in the interval $q_{\alpha} \sim\left(10^{6} \div 10^{8}\right)$, a reheating temperature $T_{R H} \simeq 10^{10} \mathrm{GeV}$ and for heavy right-handed Majorana neutrinos, $\bar{m} \simeq 10^{15} \mathrm{GeV}$, one gets

$$
\sum_{\alpha=1}^{n_{g}} \epsilon_{\alpha} \sim\left(10^{-2} \div 1\right)
$$

where the value for the ratio $n_{B} / s \simeq n_{L} / s \sim\left(10^{-11} \div 10^{-10}\right)$ given by primordial nucleosynthesis has been used. Note that the lower bound for $\sum_{\alpha=1}^{n_{g}} \epsilon_{\alpha}$ of eq. (45) strongly decreases if one slightly releases the very conservative condition for no decoherence (30). 
Moreover, since very heavy Majorana neutrinos with mass up to $10^{18} \mathrm{GeV}$ are still compatible with preheating scenario described in Ref. [5], it is interesting to observe that just increasing the value of $\bar{m}$ of one order of magnitude, by virtue of (20), (29) and (44) one reduces the lower bound for $\sum_{\alpha=1}^{n_{g}} \epsilon_{\alpha}$ of the same amount.

From the above considerations it follows that the required value for the ratio $n_{B} / s$ can be easily obtained for a wide range of the involved parameters, without imposing fine tuned conditions. In particular it is worth noticing that no particular mass degeneracy is necessary, and $C P$ violating effects of the same order of those predicted in the electroweak Standard Model, and measured in $K^{0}-\bar{K}^{0}$ system, are already sufficent to produce a baryon asymmetry of the correct order of magnitude.

\section{Conclusions}

In this paper we have considered a new scenario for the production of a primordial lepton number, based on decays of oscillating heavy Majorana neutrinos. In the framework of the preheating mechanism for a non-thermal production of massive fermions, we have stressed the possible role in leptogenesis of coherent superpositions of unstable mass eigenstate neutrinos. The mechanism is similar to the way an observable $C P$ asymmetry is produced in the neutral $B$ meson system, due to the $B^{0}-\bar{B}^{0}$ oscillation in time. In fact if the decaying neutrinos are linear superpositions of mass eigenstates $N_{i}$, the observability of the $C P$ violation is achieved if the different $N_{i}$ may interfere.

We have shown that if at least two neutrinos have small mass difference $\Delta m$, compared with their corresponding decay widths $\Gamma$, a microscopic asymmetry can be obtained at tree level in the Yukawa matrices, coupling these states to massless fermions and Higgses. The microscopic asymmetry is found to be proportional, for small mass differences to the ratio $\Delta m / \Gamma$. On the other hand, if the neutrino states have masses quite well separated, with respect to their decay widths, the interference effects we have described is vanishing and to get a non-vanishing microscopic asymmetry one should consider interference of the decay amplitudes at tree level with higher loop contributions [1], [5]-[9].

In this scenario, a crucial feature is that neutrinos are produced with a non-thermal distribution, thus the Sacharov out-of-equilibrium condition is implemented from the very 
beginning. If, as we have considered, neutrinos rapidly decay before any thermalization may occur, it is possible to avoid any wash out of the final lepton number so produced by inverse processes or scattering, provided the maximal temperature achieved during reheating is smaller than the mass of the lightest of the heavy Majorana.

It is finally worth stressing that, even using very conservative bounds on the couplings involved and Majorana masses, the order of magnitude of the microscopic asymmetries results compatible with $C P$ violation effects in the Standard Model. Actually this result holds for choices of $\Delta m$ which are not particularly fine tuned.

\section{Acknowledgements}

One of us, G. Miele, would like to thank A. Riotto for a useful discussion and valuable comments.

\section{Note added in proof}

Shortly after completing the paper, the role of neutrino instability in mixing phenomenon have been presented in a recent manuscript [19].

\section{References}

[1] M. Fukugita and T. Yanagida, Phys. Lett. 174, 45 (1986).

[2] D.J.H. Chung, E.W. Kolb and A. Riotto, Phys. Rev. D60, 063504 (1998).

[3] J. Ellis, J. Kim and D.V. Nanopoulos, Phys. Lett. B145, 181 (1984).

S. Sarkar, Rep. Prog. Phys. 59, 1493 (1996).

[4] G.F. Giudice, A. Riotto and I. Tkachev, JHEP 9908, 009 (1999).

[5] G.F. Giudice, M. Peloso, A. Riotto and I. Tkachev, JHEP 9908, 014 (1999).

[6] L. Covi, E. Roulet and F. Vissani, Phys. Lett. B384, 169 (1996).

[7] W. Buchmuller and M. Plumacher, Phys. Lett. B389, 73 (1996); Phys. Lett. B431, 354 (1998). 
[8] M. Flanz, E.A. Paschos and U. Sarkar, Phys. Lett. B345, 248 (1995);

M. Flanz, E.A. Paschos, U. Sarkar and J. Weiss, Phys. Lett. B389, 693 (1996);

M. Flanz and E.A. Paschos, Phys. Rev. D58, 113009 (1998).

[9] A. Pilaftsis, Int. J. Mod. Phys. A14, 1811 (1999).

[10] A.B. Carter and A.I. Sanda, Phys. Rev. Lett. 45, 952 (1980); Phys. Rev. D23, 1567 (1981).

I.I. Bigi and A.I. Sanda, Nucl. Phys. B193, 85 (1981).

[11] J.M. Frere, F.S. Ling, M.H.G. Tytgat and V. Van Elewyck, Phys. Rev. D60, 016005 (1999).

[12] L.A. Kofman, A.D. Linde and A.A. Starobinsky, Phys. Rev. Lett. 73, 3195 (1994).

Y. Shtanov, J. Traschen and R.H. Brandenberger, Phys. Rev. D51, 5438 (1995).

D. Boyanovsky, H.J. De Vega, R. Holman, D.S. Lee and A. Singh, Phys. Rev. D51, 4419 (1995).

[13] L.A. Kofman, A.D. Linde and A.A. Starobinsky, Phys. Rev. D56, 3258 (1997).

[14] A.D. Dolgov, D.P. Kirilova, P.B. Greene and L. Kofman, Phys. Lett. B448, 6 (1999).

J. Baacke, K. Heitmann and C. Patzold, Phys. Rev. D58, 125013 (1998).

[15] R. Kallosh, L. Kofman, A. Linde and A. Van Proeyen, hep-th/9907124 (1999).

G.F. Giudice, A. Riotto and I.Tkachev, JHEP 9911, 036 (1999).

[16] A.L. Maroto and A. Mazumdar, Phys. Rev. D59, 083510 (1999).

A.L. Maroto and A. Mazumdar, hep-ph/9904206.

[17] L. Kofman, A. Linde and A. Starobinsky, Phys. Rev. D56, 3258 (1997).

[18] L.B. Okun, Leptons and Quarks, North-Holland, Amsterdam, 1987.

[19] O. Lalakulich, E.A. Paschos and M. Flanz, hep-ph/0002021. 